EPJ manuscript No.

(will be inserted by the editor)

\title{
Disease Spreading in Structured Scale-Free Networks
}

\author{
Yamir Moreno ${ }^{1 \mathrm{a}}$ and Alexei Vázquez ${ }^{2}$ \\ ' 1 The Abdus Salam International Centre for Theoretical Physics, P.O. Box 586, 34100 Trieste, Italy \\ ' 2 International School for Advanced Studies SISSA/ISAS, via Beirut 4, 34014 Trieste, Italy
}

Received: October 24, 2018/ Revised version:

\begin{abstract}
We study the spreading of a disease on top of structured scale-free networks recently introduced. By means of numerical simulations we analyze the SIS and the SIR models. Our results show that when the connectivity fluctuations of the network are unbounded whether the epidemic threshold exists strongly depends on the initial density of infected individuals and the type of epidemiological model considered. Analytical arguments are provided in order to account for the observed behavior. We conclude that the peculiar topological features of this network and the absence of small-world properties determine the dynamics of epidemic spreading.
\end{abstract}

PACS. 89.75.-k Complex Systems - 89.75.Fb Structures and organization in complex systems - 05.70.Jk Critical point phenomena

\section{Introduction}

During the last years, there has been a burst of activity in the study of complex networks [1],2]. It has been shown that many social and natural systems [3, $1,5,6,6,8$, 9, 10, 11 can be represented as a graph where nodes represent individuals or agents and links stand for the physical ' interactions among them. Surprisingly, many of these networks share some important topological features such as small-world (SW) properties [12 and scale-free (SF) degree distributions [13], where the degree or connectivity $k$ of a node is the number of neighbors to which it is linked. Networks displaying scale-free degree distributions are very interesting not only for their relative abundance in Nature but also because of their peculiar statistical properties. In particular, the unbounded fluctuations of the connectivity distribution $P(k) \sim k^{-\gamma}(\gamma \leq 3)$ seem to be a blueprint of all real-world networks studied so far 迎, [2].

The SW and SF properties have considerable impact on the processes running on top of complex networks. The effects of the complex topological features of SF networks on the dynamics of epidemic disease spreading is perhaps one of the most interesting outcomes in the study of complex networks. Specifically, the understanding of spreading phenomena in these networks can shed light on a large number of practical problems, ranging from computer virus infections 14, 15] to epidemiology 16, 17. For instance, it has been recognized only recently [18, 19] that random SF networks are completely prone to epidemic spreading allowing the onset of large epidemics whatever

\footnotetext{
a e-mail: yamir@ictp.trieste.it
}

the spreading rate of the infection. This radical change with respect to the way a disease is spread in a regular structure is rooted in the diverging connectivity fluctuations of SF networks with $\gamma \leq 3$. Similarly, random SF networks have been shown to exhibit extremely robustness to random damages [20,21,22].

On the other hand, real networks are also characterized by degree correlations that might play a fundamental role in the functional properties of networked systems and on processes running on correlated networks. The study of networks with degree correlations and the extension of previous results obtained for random networks to correlated networks is quite recent [23, 24, 25, 26, 27, 28]. One of the models aimed to account for degree correlations was proposed recently by Klemm and Eguíluz [29] (henceforth referred to as deactivation model) where nodes can be deactivated with probability inversely proportional to their connectivity. The model turns out to be more rich than initially thought with a power law connectivity distribution $P(k) \sim k^{-\gamma}$ but with $\gamma$ laying in a quite wide interval $2 \leq \gamma \leq 4$ 30]. Additionally, it has been shown that the topology of the network is essentially linear being the graph a collection of stars of diverse degree connected as a chain by a number of local links, i.e., the graph lacks SW properties. While a threshold may exist [31] due to the peculiar topological properties of the network that makes the epidemic spreading to be dominated by the diffusion of the disease on a linear chain [30], the star-like graphs connected as a chain might also lead to new effects in the dynamics of epidemic spreading.

In this paper, we study in detail by large scale numerical simulations two paradigmatic epidemiological models, namely, the Susceptible-Infected-Susceptible (SIS) and the 
Yamir Moreno, Alexei Vázquez: Disease Spreading in Structured Scale-Free Networks

Susceptible-Infected-Removed (SIR) models on top of networks generated using the deactivation model. We found that the existence or not of an epidemic threshold for $\gamma \leq 3$ depends on the initial density of infected individuals while for values of $\gamma>3$ the epidemic threshold is recovered and it is universal, i.e., it does not depend on the initial density of infected individuals. We also provide analytical arguments in order to explain our numerical findings. Moreover, we show that the linear character of the graph determines the existence of a trivial threshold for the SIR model that can be mapped to a bond percolation problem in one dimension.

\section{Deactivation model}

Let us first summarize the main properties of the deactivation model introduced by Klemm and Eguíluz [29]. The growing dynamics of the network is defined in the following way: One starts from a fully connected graph of $m$ nodes that are set active. At successive time steps nodes are added one by one following the recipe: $(i)$ A new node is connected to all active nodes in the network; $(i i)$ one of the active nodes is selected for deactivation with probability

$$
p_{d}\left(k_{i}^{\mathrm{in}}\right)=\frac{\left[\sum_{j \in \mathcal{A}}\left(a+k_{j}^{\mathrm{in}}\right)^{-1}\right]^{-1}}{a+k_{i}^{\mathrm{in}}} ;
$$

where the sum in Eq. (11) runs over the set of active nodes $\mathcal{A}, a$ is a model parameter, and $k_{i}^{\text {in }}$ denotes the in-degree of the $i$-th node. Finally, (iii) the new node just added is set active.

Recently, it has been shown 30] that most of the topological properties (in particular, the degree distribution) of the network generated using the above rules are very sensitive to the order in which steps $($ ii $)$ and (iii) are performed. Thus we shall discriminate in the following two cases 30:

- Model A: (ii) is performed before (iii) .

- Model B: (iii) is performed after (ii).

The deactivation model is usually run taking $a=m$. This makes the deactivation probability inversely proportional to the total connectivity of the nodes $k=m+k^{\text {in }}$. The connectivity distribution can be analytically obtained for the limiting cases of lowest [30] and largest $m$ [31] for each model resulting in a power law that reads as,

$$
P(k) \sim k^{-\gamma},
$$

where the exact value of $\gamma$ depends on the model considered and the value of $m$ such that,

$$
\begin{array}{lll}
\text { - model } A \text { with } a=m & \Longrightarrow & 3<\gamma \leq 4 \\
- \text { model } B \text { with } a=m & \Longrightarrow & 2 \leq \gamma<3 .
\end{array}
$$

Noticeable and relevant for the physical processes run on top of this model, the dynamics of the deactivation model allows exponents $\gamma$ of the connectivity distribution that can lead to an unbounded second moment $\left(\left\langle k^{2}\right\rangle \rightarrow \infty\right.$ for model B) or to finite connectivity fluctuations $\left(\left\langle k^{2}\right\rangle\right.$ is finite for model A). More important, however, is the fact that the generated networks lack SW properties. Smallworld properties refer to the fact that in many complex networks one can go from one node to any other node of the system visiting a very small number of intermediate nodes. The minimum number of such intermediate nodes for each pair $(i, j)$ of nodes in the network is called the minimum path length between $i$ and $j$, and the diameter of the network is defined as the largest among the shortest paths between any two nodes in the network. For networks displaying SW properties, the graph diameter grows, at most, logarithmically with the system size $N$.

The networks generated with the deactivation model consist of a collection of star-like graphs connected as a chain, resembling the topology of a one dimensional lattice. In fact, irrespective of the version considered, both the diameter and the average minimum path distance scale linearly with the network size $N$ [30]. In particular, the mean square displacement of a random walker on the deactivation model scales with time almost as for a one dimensional lattice qualifying for a slightly subdiffusive behavior 30]. Hence, the existence of an epidemic threshold in the deactivation model is not surprising. However, at the same time, the peculiar characteristics of the graph and, in particular, the presence of stars with very large connectivities introduces new and interesting effects in the dynamics of disease spreading. As we shall discuss in the following section, whether or not a finite prevalence sets in for the SIS model would depend on the initial density of infected individuals.

\section{The SIS Model}

In the standard SIS epidemiological model [16], each node of the network represents an individual and each link is the connection along which the individuals interact and the epidemic can be transmitted. In this model, the individuals can exist in two possible states, namely, susceptible or healthy and infected. Individuals removal due to death or acquired immunization is not allowed and thus they stochastically move through the cycle susceptible $\rightarrow$ infected $\rightarrow$ susceptible. The disease transmission is described in an effective way. At each time step, each susceptible node connected to one or more infected nodes gets the infection with probability $\lambda$, while the infected nodes are cured and become again susceptible at a rate $\delta$ (henceforth set to 1 without lack of generality). In the SIS model whenever the epidemic pervades the system, it gets into an endemic state with a stationary value for the density of infected nodes that acts as the order parameter of the model.

Results on regular structures point out that there is a threshold below which the system does not reach such a stationary state and the infection dies out [16, 19]. Moreover, the value of the prevalence at the stationary state and its own existence does not depend on the initial density of infected individuals. One can start with an initial density $\rho_{0}=1 / N$ of infected individuals or from, let's say, 
half of the lattice infected (in general, $\rho_{0}=$ const): The prevalence self-organizes into a stationary state in both cases. It is worth noting that both situations are feasible in practice and thus physically relevant.

On the other hand, the behavior of the SIS model on random SF networks is radically different 18, 19 when $\gamma \leq 3$. In this case, there is no epidemic threshold in the thermodynamic limit and the networks are completely prone to the spreading of the disease. This drastic change of behavior is due to the diverging connectivity fluctuations of SF networks. Moreover, it has also been shown that for finite-size system an effective threshold is recovered, but its value is significantly overestimated [32].

In order to study the SIS model in deactivation model networks, we first generate networks using the algorithm described in the preceding section for both settings of the model A and B with $a=m=3$. Then we let the system evolve according to the SIS dynamics. Large scale numerical simulations were performed in networks of size up to $N=10^{6}$, averaging over at least 100 different realizations on at least 10 different realizations of the network. Initially, a fraction of nodes $\rho_{0}$ was infected and we let the system relax into the steady state where the prevalence $\rho$ attains its stationary value. Two initial conditions for the density of infected individuals were considered. In the first case, we start from a single infected individual, $\rho_{0}=1 / N$, and set the observation time $t_{m}$ to be $10^{6}$ time steps, that is, the state of each node is updated up to $t_{m}$ times if there is at least one infected individual. Then, we repeat the process but starting with homogeneous initial conditions. This is achieved by infecting a finite fraction of the network $\rho_{0}=$ const. and monitoring as before whether a stationary state sets in with its corresponding prevalence for the same observation time $t_{m}$. It is worth recalling that the prevalence in the stationary state is computed as the average over all surviving trials in both cases.

The results obtained are depicted in Fig. 11 where the steady state prevalence has been drawn as a function of the spreading rate $\lambda$. As expected for model A the epidemic threshold would appear again for both initial conditions since in this case together with the lack of SW properties and the linearity of the network, the second moment $\left\langle k^{2}\right\rangle$ is finite with $3<\gamma \leq 4$. In this case, the phase diagram is the same regardless of the initial density of infected individuals $\rho_{0}$. It is worth noting that whenever an epidemic threshold exists, its value seems to be the same (within statistical errors) for both versions of the deactivation model, depending only on the average connectivity $\langle k\rangle$. This implies in its turn that the dynamics of the infection spreading is mainly determined by the linear star-like structure of the graphs.

Two radically different behaviors can be noted for the deactivation model $\mathrm{B}$ depending on the initial condition. In this case the exponent of the power law decay of the degree distribution is in the interval $2 \leq \gamma<3$. In particular a best fit analysis for networks of size $N=10^{7}$ yields an exponent $\gamma=2.65 \pm 0.05$ for $m=3$. When the system evolves from a single infected individual, an epidemic threshold $\lambda_{c}$ appears, below which the epidemic
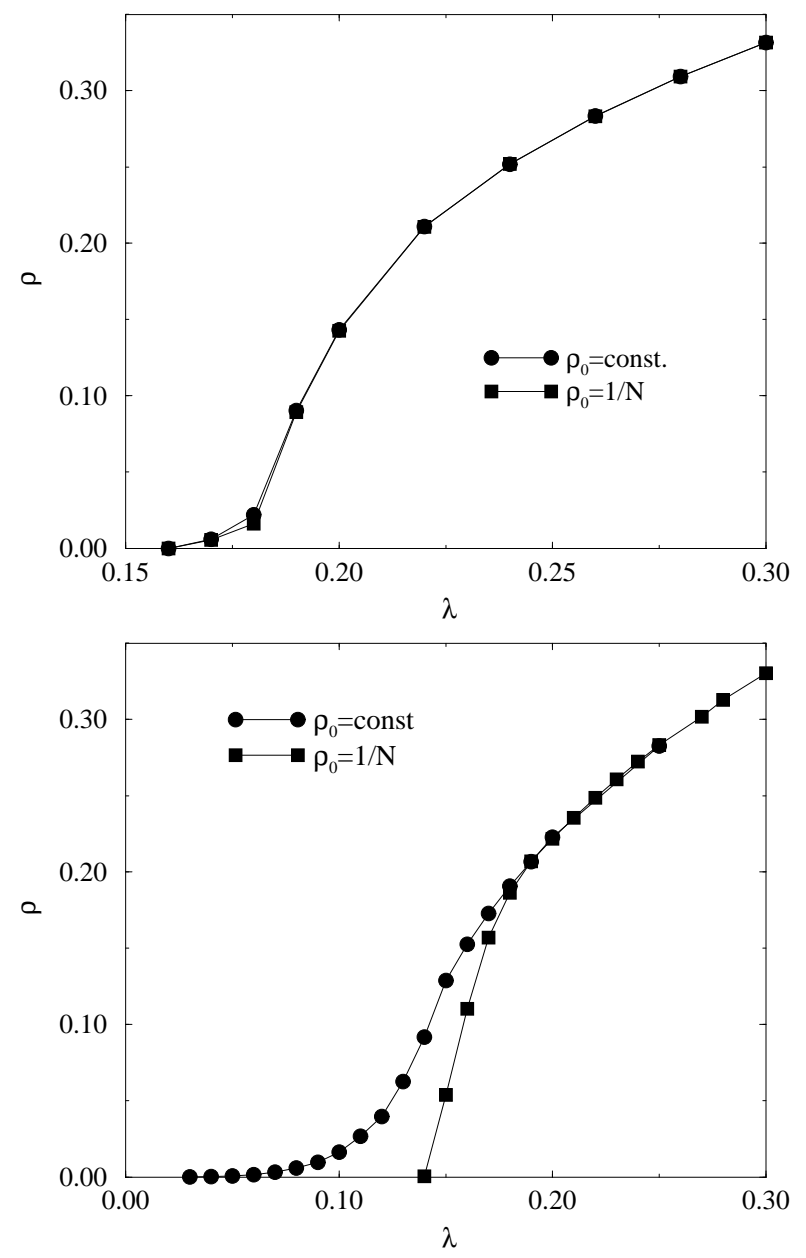

Fig. 1. Phase diagram for the deactivation model A (top) and B (bottom). The density of infected nodes $\rho$ for two initial conditions is plotted as a function of the spreading rate $\lambda$. The size of the system is $N=10^{6}$ and the average connectivity was set to $\langle k\rangle=6(m=3)$. For model A the epidemic threshold is independent of $\rho_{0}$ and is equal to $\lambda_{c}=0.16(1)$. For model B the epidemic threshold depends on $\rho_{0}$. For an initial state with a single infected individual $\lambda_{c}=0.14(1)$, whereas for homogeneous initial conditions $\lambda_{c} \rightarrow 0$ in the thermodynamic limit.

cannot pervade the network. In this case the fact that in the thermodynamic limit the connectivity fluctuations are diverging is canceled by the effects introduced by the linear topological nature of the network and the lack of SW properties. As advanced in Ref. [30], the SIS dynamics can be reduced in a coarse grained picture to the diffusion of the disease on a linear chain. Thus, the classical picture for regular structures with an epidemic threshold is recovered.

For homogeneous initial conditions, the phase diagram is completely different. In this case, the stars with high connectivity can let the epidemic survive and a final stationary state is reached by a long power-law decay. In order to rule out the presence of finite size effects hiding an abrupt transition we have checked the behavior of the stationary prevalence for several system sizes. Note that 


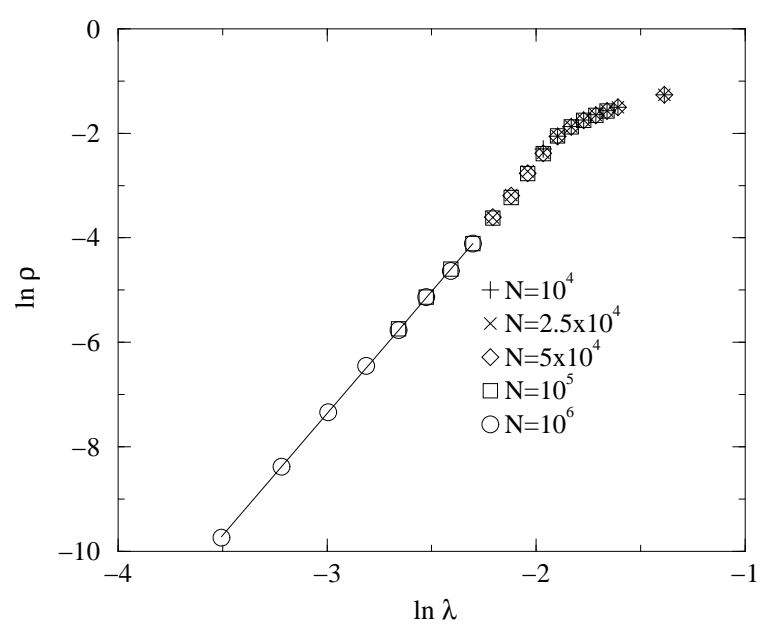

Fig. 2. Prevalence $\rho$ for the SIS model in scale-free correlated networks generated using deactivation model $\mathrm{B}$. The average connectivity of the network is in this case $\langle k\rangle=6(m=3)$. The straight line is a fit to the form $\rho(\lambda) \sim \lambda^{\beta}$, with $\beta \approx 4.5$.

larger system sizes are needed in order to correctly depict the prevalence behavior for $\lambda \ll 1$, since finite size networks induce finite size corrections to the zero threshold 18, 19. The results obtained are plotted in Fig. 2. The straight line is a fit to the form $\rho(\lambda) \sim \lambda^{\beta}$, with $\beta \approx 4.5$, showing that the infection prevalence is assuming a finite stationary value for all values $\lambda>0$. This numerical evidence confirms the previous picture obtained for uncorrelated scale-free networks, namely, the absence of an epidemic threshold in the thermodynamic limit. It is interesting to recall that using a dynamical mean field approach for uncorrelated networks with scale-free connectivity distributions given by $P(k) \sim k^{-\gamma}$, the same functional form $\rho(\lambda) \sim \lambda^{\beta}$ is predicted when $2<\gamma<3$, although in this case satisfying the relation $\beta=1 /(3-\gamma)$ [19]. Hence, it seems that correlations, while preserving the general functional form, make the prevalence to decay more faster than it does in the uncorrelated case. This in turn may explain why in finite systems the epidemic threshold is smaller than its counterpart in uncorrelated networks, a fact also observed in random network models 24. Below, we provide an analytical argument that help understand the origin of these differences by analyzing the SIS model on an ensemble of disconnected stars.

\subsection{SIS model on an ensemble of disconnected stars}

Let us study a graph made up of an ensemble of disconnected stars. To be more precise, we consider a graph where all nodes with degree $k>1$ are connected to nodes with degree $k=1$. If $P(k)(k \geq 1)$ is the degree distribution then $P(1)=\sum_{k>1} k P(k)$. Since the stars are isolated the stationary prevalence is given by

$$
\rho=\sum_{k>1} P(k) n(k) v(k)
$$

where $n(k)$ is the stationary number of infected nodes on a star of degree $k$ and $v_{k}$ is the probability that at $t=0$ there was at least one infected node in a star with $k$ leaves.

Now, to compute $n(k)$ we focus on the SIS dynamics on a single star. The spreading takes place from the central node to the leaves, then from the leaves to the central node and so on. For stars with a large number of leaves $(k \gg 1)$ we can approximate the average number of infected nodes by the number of infected leaves. Hence, the number of infected nodes at step $t+1$ is

$$
n(k, t+1)=\left[1-(1-\lambda)^{n(k, t)}\right] \lambda k .
$$

The first factor in the right hand side is the probability that the central node gets infected, receiving the disease from at least one leaf. The second factor is the number of infected leaves given that the center was infected. This linear map has always the trivial solution $n(k, t)=0$. To investigate its stability we assume $n(k, t) \ll 1$ resulting

$$
n(k, t+1)=\lambda k \ln \frac{1}{1-\lambda} n(k, t)
$$

Thus, there is a critical star size

$$
k_{c}(\lambda)=\left(\lambda \ln \frac{1}{1-\lambda}\right)^{-1},
$$

such that for $k<k_{c}$ the prefactor in the right hand side of Eq. (5) is smaller than 1 and, therefore, $n(k)=0$. On the contrary, for $k>k_{c}$ there is an exponential growth of $n(k, t)$ indicating that the solution $n(k, t)=0$ is not stable, i.e. $n(k)>0$. Moreover, the critical degree depends on $\lambda$, with the limiting cases $k_{c}(0)=\infty$ and $k_{c}(1)=0$.

Going back to Eq. (3) we obtain that

$$
\rho=\sum_{k>k_{c}} P(k) n(k) v(k) \text {. }
$$

Let us now distinguish between two different initial conditions considered before. In one case we start from only one infected node. The probability that one node of a star of $k$ leaves is infected is

$$
v^{(1)}(k)=\frac{k+1}{N}
$$

The substitution of this expression in Eq. (7) yields

$$
\rho^{(1)}=\sum_{k>k_{c}} P(k) n(k) \frac{(k+1)}{N} \sim \frac{\left\langle k^{2}\right\rangle}{N} \text {. }
$$

Now, for $2<\gamma<3$ the second moment grows at most as $\left\langle k^{2}\right\rangle=\mathcal{O}\left(N^{3-\gamma}\right)$. Hence, in the thermodynamic limit the prevalence is asymptotically zero. However, a different result is obtained if we infect a finite fraction $\rho_{0}$ of the nodes. In this case the probability that at least one node of a star with $k$ leaves is infected is given by

$$
v^{(2)}(k)=1-\left(1-\rho_{0}\right)^{k+1} \geq \rho_{0} .
$$


From this expression and Eq. (7) it follows that

$$
\rho^{(2)} \geq \rho_{0} \sum_{k>k_{c}} P(k) n(k) .
$$

Thus, there is a finite prevalence in the stationary state.

These two opposite scenarios are the same observed in the numerical simulations of the preceding section. When there is a finite fraction of infected nodes at $t=0$, a finite prevalence is obtained. On the contrary, when the initial infection is concentrated in only one node, the stationary state reflects the topological nature of the graph. For the ensemble of disconnected stars we obtain a stationary state with no infected node, corresponding to the SIS model in a zero-dimensional system. On the other hand, for the deactivation model, we get a phase transition at a finite infection rate as expected for a one-dimensional system [33].

\section{SIR model}

The SIR model assumes that individuals can exist in three classes: susceptible, infected and removed. The main difference with the SIS model is that once an individual gets infected it is removed and, therefore, it can not catch the infection again. If we start from a single infected node the size of the outbreak of the disease is given by the number of nodes that can be reached assuming that each link is occupied with a probability $\lambda$. Thus, the SIR model is equivalent to a bond percolation problem with bond occupation probability $\lambda$ [34. Moreover, the size of the outbreak is just the size of the giant component.

The study of the SIR model in random SF networks confirmed the epidemiological picture obtained for the SIS model in complex networks with power-law connectivity distributions $[35,36]$. For instance, it has been shown that the effective epidemic threshold is inversely proportional to the connectivity fluctuations $\left\langle k^{2}\right\rangle$ and hence it is vanishing in the thermodynamic limit $N \rightarrow \infty$ for $\gamma \leq 3$. The high heterogeneity of SF networks also causes that the relative incidence of an outbreak strongly depends on the connectivity of the first infected nodes [36]. This dependency on the initial seed should not be confused with the dependency on the initial conditions found previously since for the SIR model the differences in the relative incidence of an epidemic outbreak is an intrinsic effect of the large heterogeneity in the connectivity distribution of SF networks and is not related to the presence of any topological constrain.

We have made numerical simulations of the bond percolation problem on top of the structured networks generated using the two variants of the deactivation model, A and $\mathrm{B}$. Once the graphs are generated each link is removed with a probability $1-\lambda$. Then, the size of the giant component of the resulting graph is computed. All the results reported below were obtained taking an average over 10 graph realizations of the deactivation model and 10 realizations of the link removal procedure. In Fig. 3 we plot the size of the giant component $S$ as a function of $\lambda$ for
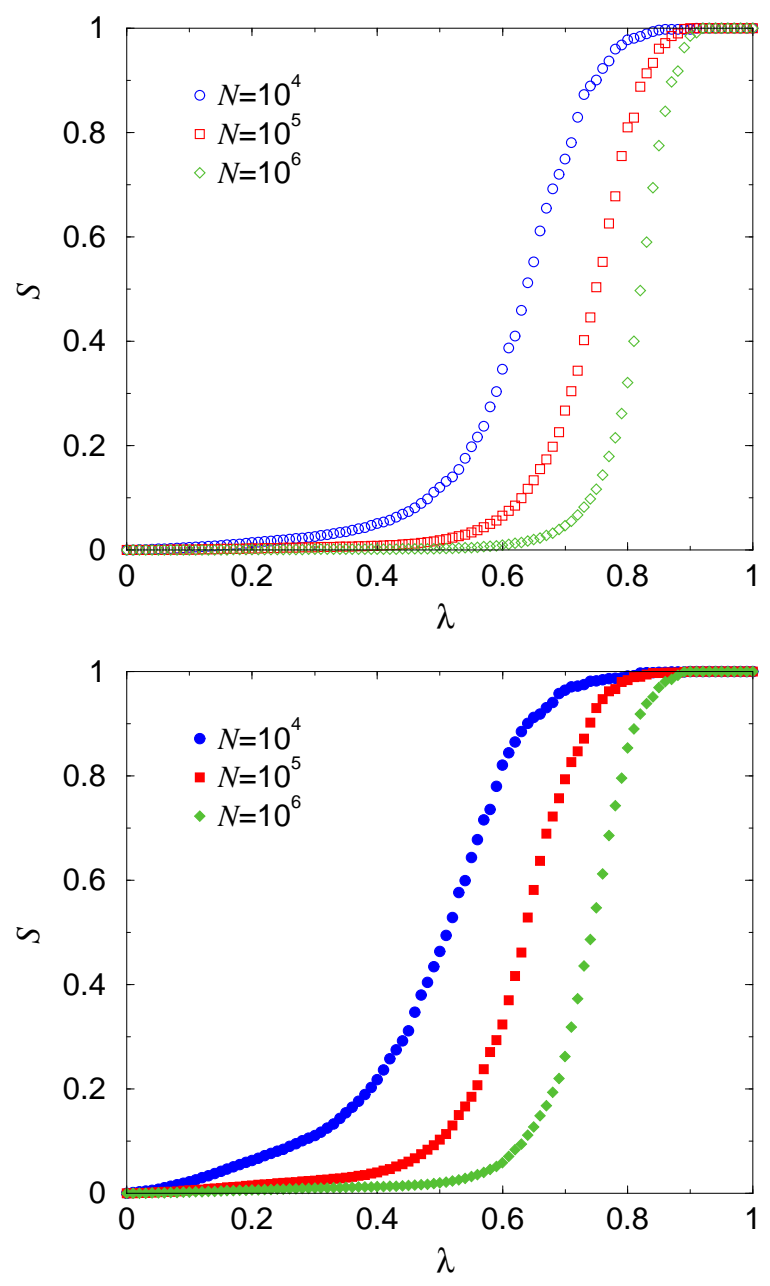

Fig. 3. Size of the giant component as a function of the bond occupation probability $\lambda$ for the network generated with $m=$ 3. The top figure (open symbols) corresponds to model A and the bottom figure (filled symbols) to model B.

different graph sizes $N$ for models A and B, with $m=3$. Notice that in both cases the qualitative picture is the same.

Between the limiting cases $S(0)=0$ and $S(1)=1$, there is an intermediate range of $\lambda$ where $S$ goes from a value close to 0 to a value close to 1 . The width of this interval decreases appreciable with increasing $N$. Moreover, the point at which the transition takes place systematically shifts to larger values of $\lambda$ approaching 1 . Since the deactivation model has essentially a one dimensional topology we expect that in the large $N$ limit $S=0$ for any $\lambda<1$. This hypothesis can not be confirmed by direct observation of Fig. 3 but it can be checked using a finite size scaling analysis. When we plot $S$ as a function $1-\lambda$ in a log-linear scale we observe equidistant shifts of the curves after increasing $N$ from $10^{4}$ to $10^{5}$ and then to $10^{6}$. This scenario corresponds to a scaling of the form $S=f\left[(1-\lambda) N^{\alpha}\right]$, where $f(x)$ is a scaling function that is independent of $N$ and $\alpha$ is a scaling exponent. 


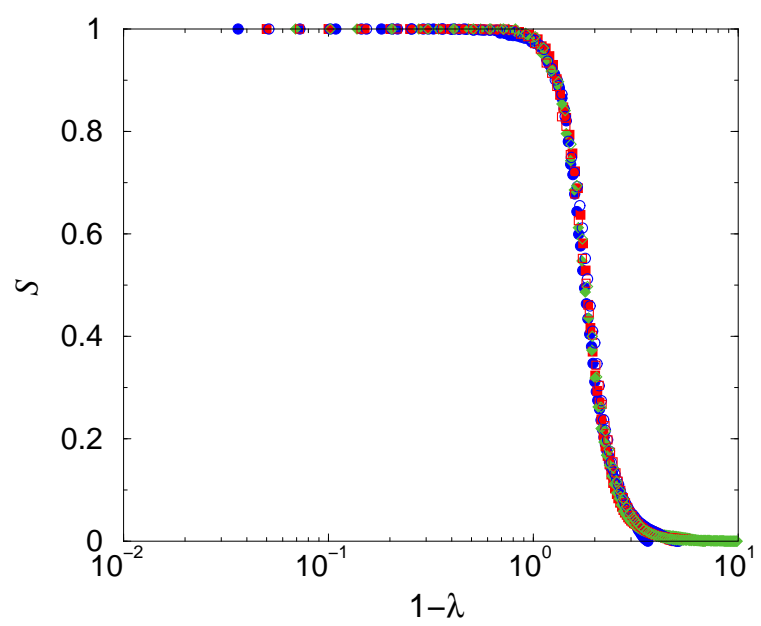

Fig. 4. Size of the giant component as a function of the rescaled variable $(1-\lambda) N^{\alpha}$, with $\alpha=0.15$ and $\alpha=0.14$ for models $\mathrm{A}$ and $\mathrm{B}$, respectively. The symbols are in correspondence with those in Fig. 3. All the curves of model A were shifted by the same amount to the right to make evident the similarity between the curves of both models.

In Fig. 1 we plot $S$ versus $(1-\lambda) N^{\alpha}$ in a log-linear scale using the value of $\alpha$ that gives the best data collapse. This was achieved for $\alpha=0.15(1)$ and $\alpha=0.14(1)$ for models A and B, respectively. Within the numerical error, the exponent $\alpha$ is the same for both models. Moreover the scaling function $f(x)$ is also unique, up to a constant factor. These results point out that with regard to the bond percolation problem, or the SIR model, the differences in the degree distributions of model $\mathrm{A}$ and $\mathrm{B}$ are qualitatively irrelevant.

\section{Discussion and conclusions}

We have study the dynamics of infectious diseases in structured scale-free networks generated using the deactivation model introduced by Klemm and Eguíluz. By means of large scale numerical simulations, we have shown that the existence or not of an epidemic threshold for the SIS model depends on the initial density of infected individuals when the connectivity fluctuations of the network are unbounded. This effect is completely new and reflects the peculiar topological nature of the networks generated with the deactivation model algorithm. Guided by the analytical solution to the SIS model in an ensemble of disconnected stars, we may summarize the SIS dynamics as follows.

The linear topological nature of the networks provoke the dynamics of the epidemic spreading to be almost purely diffusive, like in a one-dimensional lattice. However, for model B the small value of $\gamma$, that leads to the divergence of the connectivity fluctuations, allows the existence of stars with very large connectivities such that for any value of $\lambda$ the infection can always get trapped in these stars. Hence, starting from homogeneous initial conditions, the probability of hitting a star in which the epidemic survives is not zero. This is not certainly the case if one starts at a single infected node. In this case, there is no time for the infection to spread through the network. It would be of further interest to study this diffusion process in more details, for example, by looking at the distribution of waiting times for each node in the cicle infected $\rightarrow$ susceptible $\rightarrow$ infected. On the other hand, when the connectivity fluctuations are finite (model A), the probability of finding a star with degree $k>k_{c}(\lambda)$ is zero below $\lambda_{c}$ and thus the epidemic threshold is recovered for all possible initial conditions.

Finally, the study of the SIR model confirms that the dominant factor in this case is the chain structure of the network with star-like nodes connected locally. The threshold for this epidemiological model coincides, as it should, with the critical point of the corresponding one-dimensional bond percolation problem that gives an epidemic threshold $\lambda_{c}=1$.

In summary, we have provided evidences that in this kind of networks the dominant factor determining the behavior of both the SIS and the SIR models is the linear topology of the network. The existence of high degree nodes introduces new effects such as the dependency on the initial conditions for the deactivation model B with $\gamma \leq 3$. As a final remark, we should say that the results for the SIS and the SIR models here reported cannot be directly extended to all correlated networks. The peculiar topological features of the networks generated with the deactivation model make them unique within the class of correlated networks. Some recent works have begun to [25, 228, 37] address the SIS and the SIR models in correlated networks establishing the general conditions for the existence or not of an epidemic threshold. The extensive numerical exploration of these models in such networks is still to do.

We thank A. Vespignani and R. Pastor-Satorras for helpful comments and discussions. This work has been partially supported by the European Commission - Fet Open project COSIN IST-2001-33555.

\section{References}

1. A.-L. Barabási, R. Albert, Rev. Mod. Phys. 74, 42 (2002).

2. S. N. Dorogovtsev and J. F. F. Mendes, Adv. Phys. 51, 1079 (2002).

3. M. Faloutsos, P. Faloutsos, and C. Faloutsos, Comput. Commun. Rev. 29, 251 (1999).

4. R. Pastor-Satorras, A. Vázquez, and A. Vespignani, Phys. Rev. Lett. 87, 258701 (2001).

5. R. Albert, H. Jeong, and A.-L. Barabási, Nature 401, 130 (1999).

6. S. H. Strogatz, Nature 410, 268 (2001).

7. J. M. Montoya and R. V. Solé, J. Theor. Biol. 214, 405 (2002).

8. A. Wagner, Mol. Biol. Evol. 18, 1283 2001).

9. H. Jeong, S. Mason, A. L. Barabási, and Z. N. Oltvai, Nature 411, 41 (2001). 
10. R. V. Solé, R. Pastor-Satorras, E. Smith, and T. Kepler, Adv. Complex. Syst. 5, 43 (2002).

11. A. Vázquez, A. Flammini, A. Maritan, and A. Vespignani, Modellinq of protein interaction networks (2001, preprint cond-mat/0108043.

12. D. J. Watts and S. H. Strogatz, Nature 393, 440 (1998).

13. A.-L. Barabási and R. Albert, Science 286, 509 (1999).

14. M. E. J. Newman, S. Forrest, and J. Balthrop, Phys. Rev. E 66, 035101(R) (2002).

15. S. R. White, J. O. Kephart, and D. M. Chess, Computer Viruses: A Global Perspective, in Proceedings of the 5th Virus Bulletin International Conference, Boston, 1995.

16. J. D. Murray, Mathematical Biology (Springer Verlag, Berlin, 1993).

17. R. M. Anderson and R. M. May, Infectious Diseases in Humans (Oxford University Press, Oxford, 1992).

18. R. Pastor-Satorras and A. Vespignani, Phys. Rev. Lett. 86, 3200 (2001).

19. R. Pastor-Satorras and A. Vespignani, Phys. Rev. E 63, 066117 (2001).

20. R. A. Albert, H. Jeong, and A.-L. Barabási, Nature 406, 378 (2000).

21. D. S. Callaway, M. E. J. Newman, S. H. Strogatz, and D. J. Watts, Phys. Rev. Lett. 85, 5468 (2000).

22. R. Cohen, K. Erez, D. ben Avraham, and S. Havlin, Phys. Rev. Lett. 86, 3682 (2001).

23. J. Berg and M. Lässig, Correlated random networks (2002), Preprint condmat/0205589.

24. M. Boguñá and R. Pastor-Satorras, Epidemic spreading in correlated complex networks, (2002), Preprint condmat/0205621.

25. M. Boguñá, R. Pastor-Satorras, and A. Vespignani, Absence of epidemic threshold in scale-free networks with connectivity correlations (2002), Preprint cond-mat/0208163.

26. M. E. J. Newman, Assortative mixing in networks, (2002), Preprint cond-mat/0205405.

27. A. Vázquez and M. Weigt, Computational complexity arising from degree correlations in networks Preprint condmat/0207035 (2002).

28. A. Vázquez and Y. Moreno, Resilience to damage of graphs with degree correlations Preprint cond-mat/0209182 (2002).

29. K. Klemm and V. M. Eguíluz, Phys. Rev. E 65, 036123 (2002).

30. A. Vázquez M. Boguna, Y. Moreno, R. Pastor-Satorras, and A. Vespignani, Topology and correlations in structured scale-free networks, Preprint cond-mat/0209183 (2002).

31. V. M. Eguíluz and K. Klemm, Phys. Rev. Lett. 89, 108701 (2002).

32. R. Pastor-Satorras, and A. Vespignani, Phys. Rev. E 65, 035108(R) (2002).

33. J. Marro and R. Dickman, Nonequilibrium phase transitions in lattice models (Cambridge University Press, Cambridge, 1999).

34. M. E. J. Newman, Phys. Rev. E 66, 016128 (2002).

35. R. M. May and A. L. Lloyd, Phys. Rev. E 64, 066112 (2001).

36. Y. Moreno, R. Pastor-Satorras, and A. Vespignani, Eur. Phys. J. B 26, 521 (2002).

37. M. E. J. Newman, Mixing patterns in networks: Empirical results and models Preprint cond-mat/0209450 (2002). 